\title{
Coronary computed tomography angiography
}

\author{
Gilat Grunau MD PhD, Jonathon Leipsic MD
}

\section{Coronary computed tomography angiography (CTA) is a noninvasive imag- ing study that allows for the detection of coronary artery stenosis}

Contrast medium is injected intravenously, and multidetector technology is used to take an image of the heart during a single breath hold (acquisition time $\sim 8 \mathrm{~s}$ for 64 detectors). Blockade is recommended for patients with heart rates faster than 65 beats/ min to improve image quality and to reduce exposure to radiation. Because of its coronary vasodilatory effect, nitroglycerin is given sublingually to optimize image quality.

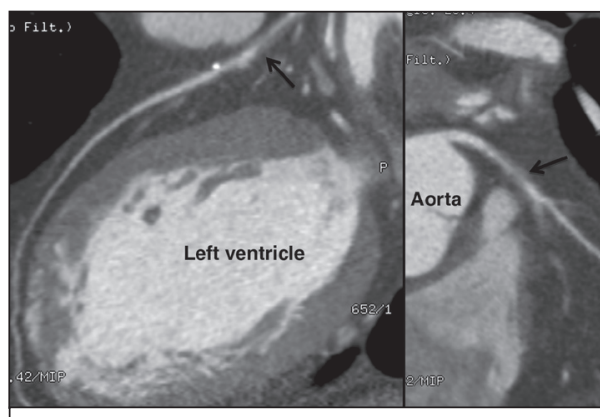

Figure 1: High-grade $(70 \%-99 \%)$ stenosis at the proximal left anterior descending artery (arrow) on the basis of predominantly noncalcified plaque.
Coronary CTA has additional advantages over invasive coronary angiography

Invasive angiography focuses only on the lumen, whereas CTA provides information on the extent and characterization of plaque. In addition, CTA allows evaluation of the course of coronary vessels, congenital abnormalities, cardiac anatomy and extracardiac pathology. Current CT machines and low-dose protocols result in a radiation dose similar to that of annual background radiation $(2-4 \mathrm{mSv}){ }^{3}$

\section{References}

1. Chang SM, Bhatti S, Nabi F. Coronary computed tomography angiography. Curr Opin Cardiol 2011;26:392402.

2. Ajlan AM, Heilbron BG, Leipsic J. Coronary computed tomography angiography for stable angina: past, present, and future. Can J Cardiol 2013;29:266-74.

3. Sun Z, Al Moudi M, Cao Y. CT angiography in the diagnosis of cardiovascular disease: a transformation in cardiovascular CT practice. Quant Imaging Med Surg 2014;4:376-96.

4. Nørgaard BL, Leipsic J, Gaur S, et al.; NXT Trial Study Group. Diagnostic performance of noninvasive fractional flow reserve derived from coronary computed tomography angiography in suspected coronary artery disease: the NXT trial. J Am Coll Cardiol 2014;63:1145-55.

5. Taylor AJ, Cerqueira M, Hodgson JM, et al. ACCF/SCCT/ACR/AHA/ASE/ASNC/ NASCI/SCAI/SCMR 2010 appropriate use criteria for cardiac computed tomography. J Am Coll Cardiol 2010;56:1864-94.
Coronary CTA has high diagnostic accuracy compared with invasive coronary angiography

Multicentre trials comparing stenosis as seen on coronary CTA with that seen on invasive coronary angiography the gold standard - have found high sensitivity $(85 \%-99 \%)$ and specificity (64\%-90\%). Coronary CTA is most useful at ruling out coronary artery disease, with a very high negative predictive value $(83 \%-99 \%){ }^{2}$

There are several indications for using coronary CTA in routine practice

Coronary CTA is useful in exercise testing when patients have intermediate risk findings or normal findings with continued symptoms; equivocal stress imaging results; or discordant results on exercise electrocardiography and stress imaging. ${ }^{5}$ Coronary CTA is not appropriate for patients with symptoms of definite acute myocardial infarction or a high pretest likelihood for coronary stenosis.

Competing interests: Jonathan Leipsic has received grant funding from GE Healthcare and Heartflow; his institution has grants pending from Edwards Life Sciences, Neovasc and Tendyne. No other competing interests were declared.

Affiliation: Department of Radiology, St. Paul's Hospital, University of British Columbia, Vancouver, BC

Correspondence to: Gilat Grunau, gilat@alumni .ubc.ca

CMAJ 2016. DOI:10.1503/cmaj.150071 